\title{
Prevalência do excesso de peso e fatores de risco para a obesidade em adultos
}

\author{
Prevalence of overweight and risk factors for obesity in adults
}

\author{
Prevalencia del exceso de peso y factores de riesgo para la obesidad en adultos
}

Tatiana Naiana Rodrigues dos Santos Porto ${ }^{1}$, Carla Lima da Rocha Cardoso'1, Luciana Stanford

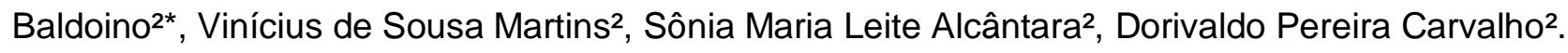

\section{RESUMO}

Objetivo: Analisar a prevalência do excesso de peso e fatores de risco para obesidade em adultos. Métodos: Trata-se de uma revisão integrativa da literatura. A coleta de dados foi de março a junho de 2017, realizada por intermédio de buscas sistemáticas utilizando os bancos de dados eletrônicos: Medline, Pubmed, Biblioteca Virtual em Saúde, a base de dados eletrônica: Scientific Electronic Library Online (SciELO) e Literatura Latino-Americana e do Caribe em Ciências da Saúde (LILACS). Resultados: O aumento de excesso de peso e obesidade foi observado maior entre indivíduos com menor nível socioeconômico de ambos os sexos. Verificou-se a baixa escolaridade como fator preponderante no aumento de peso e obesidade em adultos. Com relação ao gênero, $58 \%$ do sexo feminino e $42 \%$ do sexo masculino. Analisando esses mesmos indicadores por gênero, constatou-se que as mulheres a prevalência de sobrepeso e obesidade (I, II e III) foi de $27 \%$ e $13 \%$ com relação aos homens. Evidenciou-se a prevalência do sobrepeso e obesidade foi de $38 \%$ e $17 \%$. Considerações finais: A necessidade de políticas efetivas por meio da prevenção, promoção da saúde pelos profissionais da saúde ao público mais vulnerável ao excesso de peso e a obesidade.

Palavras-chave: Obesidade, Avaliação Nutricional, Sobrepeso, Prevalência.

\begin{abstract}
Objective: To analyze the prevalence of overweight and risk factors for obesity in adults. Methods: This is an integrative review of the literature. Data collection was from March to June 2017, carried out through systematic searches using the electronic databases: Medline, Pubmed, Virtual Health Library, electronic database: Scientific Electronic Library Online (SciELO) and Latin Literature -American and Caribbean in Health Sciences (LILACS). Results: The increase in excess weight and obesity was observed higher among individuals with lower socioeconomic status of both sexes. Low schooling was seen as a preponderant factor in weight gain and obesity in adults. Regarding gender, $58 \%$ of females and $42 \%$ of males. Analyzing these same indicators by gender, it was found that the prevalence of overweight and obesity (I, II and III) was $27 \%$ and $13 \%$ in relation to men. It was evidenced the prevalence of overweight and obesity was $38 \%$ and $17 \%$. Final considerations: The need for effective policies through prevention, health promotion by health professionals to the public more vulnerable to overweight and obesity.
\end{abstract}

Keywords: Obesity, Nutrition Evaluation, Overweight, Prevalence.

1 Enfermeira, Graduação em Enfermagem pela Faculdade IESM, Timon-MA.

2 Docentes da Faculdade IESM, Timon-MA. *E-mail: Isbaldoino@hotmail.com 


\section{RESUMEN}

Objetivo: Analizar la prevalencia del exceso de peso y factores de riesgo para la obesidad en adultos. Métodos: Se trata de una revisión integrativa de la literatura. La recolección de datos fue de marzo a junio de 2017, realizada a través de búsquedas sistemáticas utilizando los bancos de datos electrónicos: Medline, Pubmed, Biblioteca Virtual en Salud, la base de datos electrónica: Scientific Electronic Library Online (SciELO) y Literatura Latino-Americana y del Caribe en Ciencias de la Salud (LILACS). Resultados: El aumento de exceso de peso y obesidad fue observado mayor entre individuos con menor nivel socioeconómico de ambos sexos. Se verificó la baja escolaridad como factor preponderante en el aumento de peso y obesidad en adultos. Con respecto al género, el $58 \%$ del sexo femenino y el $42 \%$ del sexo masculino. En el análisis de estos mismos indicadores por género, se constató que las mujeres la prevalencia de sobrepeso y obesidad (I, II y III) fue del $27 \%$ y el $13 \%$ con relación a los hombres. Se evidenció la prevalencia del sobrepeso y obesidad fue de $38 \%$ y $17 \%$. Consideraciones finales: La necesidad de políticas efectivas por medio de la prevención, promoción de la salud por los profesionales de la salud al público más vulnerable al exceso de peso y la obesidad.

Palabras-clave: Obesidad, Evaluación Nutricional, Sobrepeso, Prevalencia.

\section{INTRODUÇÃO}

A obesidade é definida pelo excesso de gordura corporal, ligada a vários riscos para a saúde, abrangendo os aspectos sociais, comportamentais e biológicos. A partir desse excesso de gordura é possível identificar a predisposição para doenças crônicas não transmissíveis, como por exemplo, o diabetes, as doenças cardiovasculares e câncer (ALMEIDA LM, et al., 2017).

No Brasil, segundo dados da última pesquisa de orçamentos familiares realizada, cerca de $15 \%$ dos adultos apresentavam obesidade e cerca de metade da população maior de 20 anos apresentava excesso de peso (BRASIL, 2014).

Segundo Miranda JMQ, et al. (2015), nestas duas últimas décadas, a obesidade assumiu proporções alarmantes no mundo inteiro, paralelamente ao aumento do sedentarismo e ao fácil acesso a alimentos altamente palatáveis e hipercalóricos como os chamados fastfood. Este aumento de prevalência verifica-se em todas as faixas etárias, sendo que a obesidade na adolescência constitui um dos mais importantes problemas de saúde pública, não só pela possibilidade de manutenção dessa condição até a vida adulta como também por aumentar o risco para surgimento precoce das complicações metabólicas associadas ao excesso de gordura corporal, nomeadamente a síndrome metabólica.

Entre as principais causas da obesidade, pode-se citar uma alimentação inadequada com uma grande quantidade de carboidratos e açucares pouco consumo de frutas e o sedentarismo. Fatores genéticos também podem influenciar no ganho de peso e na dificuldade de emagrecimento. Portanto com medidas simples como uma atividade física moderada e uma alimentação balanceada, pode-se reduzir drasticamente o excesso de gordura corporal (SHOLER MC, et al., 2016).

Segundo Teixeira RC, et al. (2016), a obesidade também possui fatores genéticos, os quais por meio de um rastreamento genético pode-se indicar quais filhos com pais obesos terá um maior risco de se tornarem também obesos. Apesar disso, a explicação da genética é extremamente difícil, visto que pais e filhos, além da semelhança dos genes, costumam também compartilhar hábitos semelhantes de alimentação e de atividade física.

Há diversos métodos para avaliar se o peso de uma pessoa está dentro da faixa considerada adequada. Na prática clínica cotidiana e para a avaliação em nível populacional, recomenda-se o uso do Índice de Massa Corporal (IMC), o IMC é avaliado através do cálculo de peso sobre a altura, por sua facilidade de mensuração e por ser uma medida não invasiva e de baixo custo (BRASIL, 2014). 
De acordo com Schommer AV, et al. (2013), é importante o conhecimento do excesso de peso corporal e da adiposidade central no risco para a saúde, para tal faz-se o uso do índice de massa corporal (IMC) onde ele é utilizado como um indicador de excesso de peso global, enquanto a circunferência abdominal (CA) tem sido utilizada como indicador de obesidade central e pode ter relação com o desenvolvimento das doenças cardiovasculares, do diabetes melito tipo 1 e também da morte prematura. A CA é o melhor indicador antropométrico na detecção dos fatores de risco cardiovascular em adolescentes e adultos jovens, incluindo níveis arteriais aumentados e sensibilidade à insulina.

Na concepção de Bravin BM, et al. (2015), a obesidade apresenta-se como um risco para a saúde, com o fato de contribuir para o desenvolvimento das doenças já citadas, contribui também para o desenvolvimento de hipercolesterolemia, síndrome de apneia obstrutiva do sono e afecções osteomioarticulares, a obesidade causa outros prejuízos além de doenças cardiovasculares. Entre as complicações oriundas da obesidade, destacam-se aquelas relacionadas à síndrome metabólica, no entanto, outras alterações, como problemas respiratórios, dermatológicos e ortopédicos, como alterações posturais, dores musculoesqueléticas e doenças como a epifisiólise da cabeça femoral, as osteocondrites e a tíbia vara, também podem ocorrer.

Para Freire RS, et al. (2014) a prática da educação física é de suma importância em qualquer idade e tem sido considerado um meio de preservar a saúde e melhorar a qualidade de vida do ser humano. $O$ aumento da inatividade física tem relação com as mudanças no estilo de vida das pessoas, associadas às facilidades da vida moderna, como a utilização de automóveis, escadas rolantes e pelo lazer hipocinético, como assistir televisão, usar computadores e vídeo games. O baixo nível de AF é considerado importante fator de risco para as doenças crônicas não transmissíveis. A carga de doenças crônicas não transmissíveis atingiu um patamar preocupante para a saúde pública. Dessa forma, a relevância da implantação de políticas públicas que estimulem a prática de AF nos grupos populacionais que são mais afetados pelo sedentarismo: os adultos, em especial as mulheres e aqueles com menor nível socioeconômico.

Considerando a alta incidência de sobrepeso e obesidade neste grupo, justifica-se a investigação detalhada de informações contidas na literatura já existente. Sendo assim a determinação do estado nutricional prevalente e dos fatores associados fornecerão importantes dados que servirão como subsídio para a formulação de estratégias preventivas. Diante do exposto, o presente estudo teve como objetivo analisar a prevalência do excesso de peso e fatores de risco para obesidade em adultos.

\section{MÉTODOS}

Trata-se de uma revisão integrativa da literatura. A coleta de dados ocorreu no período de março a junho de 2017, realizada por intermédio de buscas sistemáticas utilizando os bancos de dados eletrônicos: Medline, Pubmed, Biblioteca Virtual em Saúde, a base de dados eletrônica Scientific Electronic Library Online (SciELO) e Literatura Latino-Americana e do Caribe em Ciências da Saúde (LILACS), com os seguintes descritores: Obesidade, Avaliação nutricional, Sobrepeso e Prevalência. Para restringir a busca de dados neste estudo, foram utilizados os Operadores Booleanos (Quadro 1).

Quadro 1 - Estratégia de Busca utilizando Operadores Booleanos.

\begin{tabular}{|l|l|l|l|l|}
\hline Estado & AND & Nutricional & AND & Adultos \\
\hline Sobrepeso & OR & Obesidade & AND & Adultos \\
\hline Obesidade & AND & Adultos & \\
\hline Sobrepeso & AND & Adultos & \\
\hline Prevalência & AND & Obesidade & \\
\hline
\end{tabular}

Fonte: Sistema de Biblioteca, PUC-Rio, 2009. 
Os critérios de inclusão foram artigos publicados com o ano de publicação de 2013 a 2017, disponíveis em inglês e português e que discorriam sobre o excesso de peso e obesidade em adultos. Já como critério de exclusão optou-se por não utilizar textos incompletos e artigos que não estivessem disponíveis na íntegra on-line. Os artigos selecionados foram catalogados em instrumento previamente preenchido.

Na Tabela 1 abaixo mostra a aplicação dos critérios de inclusão e exclusão, e os artigos selecionados.

Tabela 1 - Artigos que tratam da prevalência do sobrepeso e/ou obesidade em Adultos.

\begin{tabular}{|c|c|c|c|c|}
\hline Base de Dados & $\begin{array}{c}\text { Artigos } \\
\text { encontrados }\end{array}$ & $\begin{array}{c}\text { Artigos pré- } \\
\text { selecionados }^{1}\end{array}$ & $\begin{array}{c}\text { Artigos } \\
\text { selecionados }\end{array}$ & $\begin{array}{c}\text { Artigos } \\
\text { inclú́dos }\end{array}$ \\
\hline Lilacs & 2.673 & 142 & 42 & 3 \\
\hline Scielo & 308 & 43 & 13 & 4 \\
\hline Medline & 2.866 & 23 & 11 & 2 \\
\hline Pubmed & 5 & 2 & 2 & 1 \\
\hline TOTAL & 5.852 & 210 & 68 & 10 \\
\hline
\end{tabular}

${ }^{1}$ Artigos duplicados nas bases de dados e que abordavam sobrepeso e/ou obesidade em outras faixas etárias.

${ }^{2}$ Artigos não disponíveis na íntegra on-line e Artigos de revisão.

Fonte: Pesquisa realizada no período de março a junho de 2017.

Por se tratar de base de dados públicos não houve necessidade de encaminhar essa pesquisa ao Comitê de Ética e de Pesquisa (CEP) por não haver à necessidade de participação de seres humanos de acordo com a resolução no 466/12.

\section{RESULTADOS E DISCUSSÃO}

Realizou-se a leitura do título e do resumo, sendo excluídos aqueles que não abordavam a questão norteadora da pesquisa, os que estavam duplicados nas bases sendo escolhidos em apenas uma base e excluídos nas outras, e os que não forneciam acesso ao texto completo. Após a primeira seleção, foi realizada leitura completa dos artigos. Sendo assim, a amostra final foi composta por dez artigos.

A avaliação das evidências encontradas ocorreu por meio da leitura cuidadosa dos artigos e do fichamento individual dos mesmos por meio de um instrumento previamente construído, com as informações de interesse dos pesquisadores, tais como: Título do artigo, Periódico e ano de publicação, Tipo de estudo e metodologia, População do estudo, Síntese dos resultados, Qualis Capes e Nível de evidência (Quadro 2). O nível de evidência dos estudos catalogados foram categorizados de acordo com o Oxford Centre Medicine - Based Evidence.

Portanto, parte dos dados foi apresentada em quadro esquemático, refletindo a síntese das características dos estudos analisados e outra parte foi apresentada de forma descritiva para um melhor entendimento do conteúdo dos artigos. Seguiu-se a análise e discussão dos resultados fundamentada no diálogo com os autores que discutem a temática. 
Quadro 2 - Caracterização segundo: autor/titulação, periódico de publicação e ano, tipo de estudo/metodologia, população do estudo, síntese dos resultados, qualis capes e nível de evidência. Timon, 2017.

\begin{tabular}{|c|c|c|c|c|c|c|c|}
\hline Título do estudo & Autor/Titulação & $\begin{array}{lr}\text { Períodico } & \text { de } \\
\text { publicação / } & \text { ano }\end{array}$ & $\begin{array}{lr}\text { Tipo } & \text { de } \\
\text { estudo/metodologia }\end{array}$ & $\begin{array}{l}\text { População } \\
\text { do estudo }\end{array}$ & Síntese dos resultados & $\begin{array}{l}\text { Qu } \\
\text { alis }\end{array}$ & $\begin{array}{l}\text { Nível de } \\
\text { evidência }\end{array}$ \\
\hline $\begin{array}{l}\text { Evolução do excesso } \\
\text { de peso e obesidade } \\
\text { até a idade adulta, } \\
\text { Pelotas, Rio Grande do } \\
\text { Sul, Brasil, 1982-2012 }\end{array}$ & $\begin{array}{l}\text { LIMA et al./ } \\
\text { Especialista }\end{array}$ & $\begin{array}{l}\text { Cad. Saúde Pública/ } \\
2015\end{array}$ & $\begin{array}{l}\text { Estudo de Coorte de } \\
\text { Nascimentos }\end{array}$ & $\begin{array}{l}\text { Indivíduos } \\
\text { nascidos } \\
\text { em } 1982\end{array}$ & $\begin{array}{l}\text { A prevalência de excesso de peso } \\
\text { entre os } 15 \text { e os } 30 \text { anos } \\
\text { aumentou de } 23,2 \% \text { para } 57,6 \% \text {, } \\
\text { enquanto para obesidade } 0 \\
\text { incremento foi de } 7,1 \% \text { para } 23 \% \text {. }\end{array}$ & $\mathrm{A} 2$ & $2 \mathrm{~B}$ \\
\hline 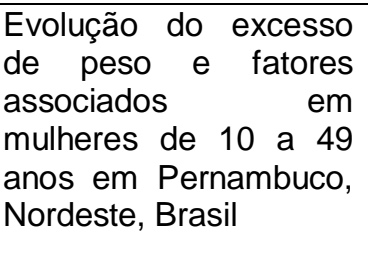 & $\begin{array}{l}\text { COSTA et al./ } \\
\text { Doutora }\end{array}$ & Rev. Nutr/ 2014 & $\begin{array}{l}\text { Estudo Transversal, } \\
\text { de base populacional }\end{array}$ & $\begin{array}{l}\text { Mulheres } \\
\text { entre } 10 \text { e } \\
19 \text { anos }\end{array}$ & $\begin{array}{l}\text { Em relação ao estado nutricional } \\
\text { no período compreendido entre } \\
1997 \text { e } 2006 \text {, houve um aumento } \\
\text { significativo no sobrepeso e } \\
\text { obesidade, com estabilização na } \\
\text { prevalência de déficit de peso. }\end{array}$ & B2 & $2 \mathrm{~B}$ \\
\hline $\begin{array}{lr}\begin{array}{l}\text { Associação } \\
\text { fatores de }\end{array} & \begin{array}{r}\text { entre } \\
\text { risco }\end{array} \\
\text { cardiovascular } & \text { e } \\
\text { indicadores } & \\
\text { antropométricos } & \text { de } \\
\text { obesidade } & \text { em } \\
\text { universitários de } & \text { São } \\
\text { Luís, Maranhão, Brasil }\end{array}$ & $\begin{array}{l}\text { CARVALHO et } \\
\text { al./ Especialista }\end{array}$ & $\begin{array}{l}\text { Ciência \& Saúde } \\
\text { Coletiva/ } 2015\end{array}$ & Estudo Transversal & $\begin{array}{l}\text { Estudantes } \\
\text { Universitário } \\
\text { s }\end{array}$ & $\begin{array}{l}\text { O sedentarismo foi bastante } \\
\text { elevado em ambos os sexos, } \\
\text { sendo maior em mulheres } \\
(73,9 \%) \text {. }\end{array}$ & $\mathrm{A} 2$ & $3 B$ \\
\hline
\end{tabular}

REAS/EJCH | Vol. Sup.22 | e308 | DOI: https://doi.org/10.25248/reas.e308.2019 Página $\mathbf{5}$ de 12 


\begin{tabular}{|c|c|c|c|c|c|c|c|}
\hline $\begin{array}{l}\text { Excesso de peso de } \\
\text { adultos residentes em } \\
\text { um aglomerado urbano } \\
\text { subnormal }\end{array}$ & $\begin{array}{l}\text { MELO, FILHO, } \\
\text { RISSIN/ } \\
\text { Pesquisadores }\end{array}$ & $\begin{array}{l}\text { Rev Bras Promoç } \\
\text { Saúde/ } 2015\end{array}$ & $\begin{array}{l}\text { Estudo observacional, } \\
\text { de caráter } \\
\text { transversal }\end{array}$ & 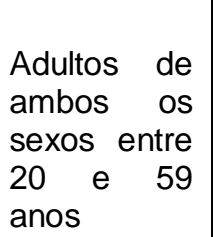 & $\begin{array}{l}\text { Em termos de etnias, } \\
59,6 \%(n=65) \text { da cor branca tinha } \\
\text { sobrepeso/obesidade, } \\
\text { condição que prevalecia para } \\
68,1 \%(n=64) \text { da cor preta }\end{array}$ & B3 & $2 \mathrm{C}$ \\
\hline $\begin{array}{l}\text { Limitantes da evolução } \\
\text { ponderal de mulheres } \\
\text { com } \\
\text { excesso de peso após } \\
\text { intervenção nutricional }\end{array}$ & $\begin{array}{l}\text { LOPES et al./ } \\
\text { Pesquisadores }\end{array}$ & $\begin{array}{l}\text { Mundo da Saúde/ } \\
2016\end{array}$ & Estudo retrospectivo & Mulheres & $\begin{array}{l}\text { O insucesso } \\
\text { da redução ponderal pode estar } \\
\text { relacionado a aspectos que } \\
\text { extrapolam a adoção de uma } \\
\text { alimentação adequada, } \\
\text { perpassando por fatores como } \\
\text { sono, sedentarismo e presença } \\
\text { de comorbidades }\end{array}$ & B2 & 4 \\
\hline $\begin{array}{l}\text { Evolução anual da } \\
\text { prevalência de } \\
\text { excesso de peso e } \\
\text { obesidade em adultos } \\
\text { nas capitais dos } 26 \\
\text { estados brasileiros e no } \\
\text { Distrito Federal entre } \\
2006 \text { e } 2012\end{array}$ & $\begin{array}{l}\text { MALTA et al/ } \\
\text { Pesquisadores }\end{array}$ & $\begin{array}{l}\text { Rev Bras Epidemiol } \\
\text { Suppl pense/ } 2014\end{array}$ & $\begin{array}{l}\text { Estudo } \\
\text { epidemiológico } \\
\text { quantitativo }\end{array}$ & $\begin{array}{l}\text { Sistema de } \\
\text { Informação } \\
\text { VIGITEL }\end{array}$ & $\begin{array}{l}\text { A prevalência de excesso de peso } \\
\text { na população adulta das } 27 \\
\text { cidades monitoradas pelo } \\
\text { VIGITEL aumentou de } 43,2 \% \\
\text { (2006) para } 51,0 \%(2012) \text {, sendo } \\
\text { de } 1,37 \% \text { o incremento anual } \\
\text { médio calculado para o período. A } \\
\text { prevalência da obesidade } \\
\text { aumentou de } 11,6 \% \text { para } 17,4 \% \text {, } \\
\text { com incremento anual de } 0,89 \% \text {. }\end{array}$ & B1 & 4 \\
\hline
\end{tabular}




\begin{tabular}{|c|c|c|c|c|c|c|c|}
\hline $\begin{array}{l}\text { Prevalência e fatores } \\
\text { associados ao excesso } \\
\text { de peso em uma } \\
\text { população urbana do } \\
\text { interior } \\
\text { do Amazonas, Brasil }\end{array}$ & $\begin{array}{l}\text { CORDEIRO, } \\
\text { FREITAS/ } \\
\text { Pesquisadoras }\end{array}$ & $\begin{array}{l}\text { Rev Bras Promoç } \\
\text { Saúde/ } 2016\end{array}$ & $\begin{array}{l}\text { Estudo descritivo, } \\
\text { transversal, de base } \\
\text { populacional }\end{array}$ & $\begin{array}{l}\text { Adultos de } \\
\text { ambos os } \\
\text { sexos > } 18 \\
\text { anos }\end{array}$ & $\begin{array}{l}\text { A maioria dos entrevistados } \\
(51,8 \%, n=231) \text { tinha mais de } 36 \\
\text { anos de idade e ensino } \\
\text { superior }(42,9 \%, n=189) \text {. Quanto } \\
\text { à renda familiar, } 49,5 \% \\
(\mathrm{n}=218) \text { dos entrevistados } \\
\text { informaram rendimento } \\
\text { mensal entre } 3 \text { e } 5 \text { salários } \\
\text { mínimos. }\end{array}$ & B3 & $2 \mathrm{~B}$ \\
\hline $\begin{array}{l}\text { Prevalência de } \\
\text { sobrepeso e obesidade } \\
\text { em discentes de uma } \\
\text { instituição de } \\
\text { ensino superior da } \\
\text { região macropolitana } \\
\text { paulista }\end{array}$ & $\begin{array}{l}\text { GUERRA et al./ } \\
\text { Mestre }\end{array}$ & $\begin{array}{l}\text { Saúde em Foco/ } \\
2016\end{array}$ & $\begin{array}{l}\text { Estudo quantitativo } \\
\text { exploratório } \\
\text { transversal }\end{array}$ & $\begin{array}{l}\text { Alunos } \\
\text { universitário } \\
\text { s }\end{array}$ & $\begin{array}{l}\text { Dentre os entrevistados, } 58 \text { \% } \\
\text { são sedentários, } 5 \% \text { não } \\
\text { consomem } \\
\text { dietas ricas em gorduras e } \\
\text { açucares, } 23 \% \text { desconhecem as } \\
\text { doenças associadas à obesidade } \\
\text { e } 72 \% \text { gostariam de participar de } \\
72 \text { programa de promoção de } \\
\text { um paúde. }\end{array}$ & B2 & $2 C$ \\
\hline $\begin{array}{l}\text { Prevalência e fatores } \\
\text { associados com } \\
\text { hipertensão arterial } \\
\text { autorreferida em } \\
\text { adultos brasileiros }\end{array}$ & $\begin{array}{l}\text { MALTA et al./ } \\
\text { Enfermeira }\end{array}$ & $\begin{array}{l}\text { Rev Saude Publica/ } \\
2017\end{array}$ & $\begin{array}{l}\text { Estudo } \\
\text { epidemiológico } \\
\text { quantitativo }\end{array}$ & $\begin{array}{l}\text { Sistema de } \\
\text { Informação } \\
\text { VIGITEL }\end{array}$ & $\begin{array}{l}\text { Prevalência de hipertensão } \\
\text { arterial autorreferida entre os } \\
\text { adultos residentes } \\
\text { nas capitais brasileiras e Distrito } \\
\text { Federal foi de } 24,1 \% \text {. Foram } \\
\text { identificadas as seguintes } \\
\text { associações com hipertensão } \\
\text { arterial autorreferida: faixa etária, } \\
\text { tomando } 18 \text { a } 24 \text { anos como } \\
\text { referência }\end{array}$ & B1 & 4 \\
\hline
\end{tabular}

REAS/EJCH | Vol. Sup.22 | e308 | DOI: https://doi.org/10.25248/reas.e308.2019 Página 7 de 12 


\begin{tabular}{|c|c|c|c|c|c|c|c|}
\hline $\begin{array}{l}\text { Associação Entre a } \\
\text { Obesidade Central e a } \\
\text { Incidência de Doenças } \\
\text { e Fatores de Risco } \\
\text { Cardiovascular }\end{array}$ & $\begin{array}{l}\text { BARROSO et al./ } \\
\text { Especialista }\end{array}$ & $\begin{array}{l}\text { International Journal } \\
\text { of Cardiovascular } \\
\text { Sciences/ } 2017\end{array}$ & Estudo transversal & $\begin{array}{ll}\text { Adultos } & \text { de } \\
\text { ambos } & \text { os } \\
\text { sexos } & \end{array}$ & 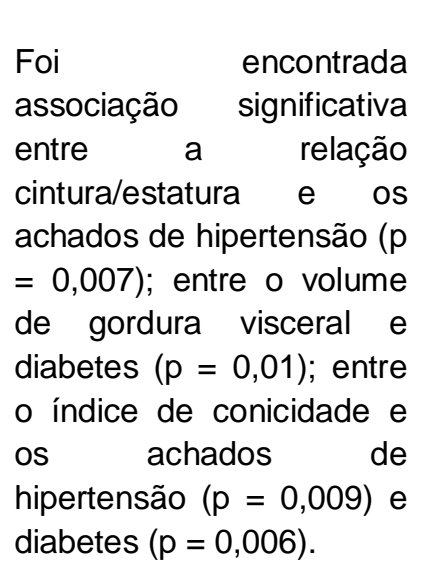 & $\mathrm{A} 2$ & $2 \mathrm{~B}$ \\
\hline
\end{tabular}


Neste estudo foi possível perceber um crescente número de estudos voltados para o público adulto jovem, visto que esta condição está comumente ligada ao público infanto-juvenil, todavia os hábitos adquiridos na infância, na maioria das vezes se refletem na vida adulta, daí a importância de estudos voltados para esta faixa etária.

Segundo Lima NP, et al. (2015) que analisaram a prevalência de excesso de peso e obesidade na adolescência e idade adulta em indivíduos, mostrou que nas mulheres, a prevalência de excesso de peso aumentou de $23,6 \%$ aos 15 anos para $52,4 \%$ aos 30 anos, enquanto para a obesidade aumentou de $6,6 \%$ para $23,8 \%$. Nos homens, o excesso de peso passou de $22,9 \%$ para $62,9 \%$, enquanto para a obesidade o crescimento foi de $7,5 \%$ para $22,1 \%$. No entanto, o aumento de excesso de peso e obesidade mostrou-se maior entre indivíduos com menor nível socioeconômico em ambos os sexos. A falta de informação sobre uma alimentação saudável e também o valor monetário deste tipo de alimento, acaba por dificultar o acesso do público mais humilde a este tipo de alimentação, contribuindo assim para o sobrepeso nestes indivíduos.

O excesso de peso aumentou de 32,8\% em 1997 para 41,7\% em 2006, apresentando um crescimento de $27,1 \%$. As mulheres da Região Metropolitana do Recife, do Interior Urbano e Rural constatou-se um aumento do excesso de peso de $40,2 \%, 6,8 \%$ e $45,2 \%$, respectivamente. Na avaliação dos fatores associados a essa patologia, em 2006, a baixa escolaridade (ensino fundamental), o baixo nível socioeconômico, se mostraram relevantes. A baixa escolaridade mostrouse importante fator no aumento de peso e obesidade em adultos, visto que a falta de informação e de conhecimento do valor nutricional dos alimentos, acaba levando-os a adotarem hábitos prejudiciais de alimentação, e abandono do hábito de práticas esportivas como formas de prevenção e de tratamento (COSTA EC, et al., 2014).

Em estudo realizado no Nordeste para avaliar a associação entre os fatores de risco cardiovascular e indicadores antropométricos em amostra de base populacional de universitários. Verificou-se que a prevalência de hipertensão foi maior em homens (57,0\% vs. $13,1 \%)$, todavia, esteve associada a maiores valores para todas as variáveis antropométricas em ambos os sexos. Evidenciou-se que o sedentarismo como fator preponderante, ficando bastante elevado em ambos os sexos, sendo maior em mulheres $(73,9 \%)$. A prevalência de HAS encontrada foi significativamente maior em homens (CARVALHO CA, et al., 2015).

Para Freire RS, et al. (2014), o sedentarismo é o fator de risco, que pode ser facilmente eliminado, visto que até mesmo uma simples caminhada pode influenciar de forma positiva na vida de um indivíduo com excesso de peso e/ou obesidade, porém é o fator que mais se encontra resistência por parte deste público, seja por falta de tempo, de interesse, e principalmente por falta de apoio por parte das pessoas do convívio social e pessoal. Vale enfocar que a prática de exercícios físicos é a primeira forma de prevenção desta condição, seguida pela alimentação saudável, são fatores que aliados produzem melhora importante neste estado.

Melo TF e Melo TL (2015) avaliaram a prevalência de excesso de peso e possíveis fatores de risco em adultos residentes em um aglomerado urbano subnormal, e a prevalência de excesso de peso foi de $62,5 \%$ ( $n=364)$, sendo maior nas mulheres $(66,1 \% ; n=251)$ do que nos homens $(56,0 \%$; $\mathrm{n}=113$ ), com um aumento progressivo até a faixa etária de 40 a 49 anos, passando a diminuir a partir daí. Nessa faixa, houve um risco de excesso de peso de 2,6 vezes. Além da idade, pertencer ao sexo feminino e não ter nenhuma ocupação também representou condições de risco. 
No Brasil em 2008/2009, foi constatado que 1,55 milhões de adultos apresentavam obesidade mórbida, totalizando $0,81 \%$ da população do país, com o índice maior na Região Sul, nas mulheres e em pessoas de cor negra. Em 2011, os custos atribuídos à obesidade totalizaram cerca de $R \$ 487,98$ milhões representando $1,9 \%$ dos gastos com assistência à saúde. Os custos da obesidade mórbida representaram $23,8 \%$ dos custos da obesidade ( $R \$ 116,2$ milhões), apesar de sua prevalência ser 18 vezes menor. Os custos com a cirurgia bariátrica no Brasil foram de $R \$ 31,5$ milhões. A obesidade mórbida teve seu custo 4,3 vezes maior do que o da obesidade (MAZUR, NAVARRO, 2015).

Em estudo de Lopes MS, et al. (2016) realizado com mulheres com excesso de peso após um ano de acompanhamento nutricional individual mensal. Dos 70 participantes, 62,9\% $(n=44)$ evoluíram insatisfatoriamente, apresentando média de $52,6 \pm 11,9$ anos e renda per capita mensal de $63,6 \%$ do salário mínimo em vigor (18,6\%; 198,3\%), características semelhantes às das mulheres com evolução satisfatória. Usuárias com evolução insatisfatória mostraram, no início do acompanhamento, maior prevalência de hipertensão arterial, dormiam menos horas por noite, assistiam televisão e/ou utilizavam computador por mais tempo, além de relataram menor sudorese durante a prática de atividade física.

É pertinente salientar que a prevalência de diabetes aumentou significativamente na população estudada, de 5,5\% para 8,0\% entre 2006 e 2014 (0,26 pontos percentuais (pp/ano). Os indivíduos com diabetes apresentaram menor frequência de comportamentos de risco e maior frequência de comportamentos de proteção quando comparados a população sem diabetes. O que está diretamente relacionada aos maus hábitos alimentares, o grande consumo de carboidratos e gorduras, o que acaba por aumentar a ocorrência de HAS e diabetes. O aumento de peso está relacionado com 0 consumo de refrigerantes e bebidas com alto teor de açúcar $(9,5 \%$ vs $25,0 \%)$, de bebida alcoólica $(8,7 \%$ vs $17,7 \%)$ e de leite com teor integral de gordura ( $44,7 \%$ vs $55,8 \%)$, enquanto as menores foram verificadas para o consumo regular de frutas e verduras $(40,8 \%$ vs $33,4 \%)$ e de carnes com gordura $(24,0 \%$ vs $32,2 \%)$.

Em ambos os grupos ainda apresentaram alta frequência de comportamentos negativos como o consumo de leite com teor integral de gordura ou refrigerantes e bebidas açucaradas e prática insuficiente de atividade física (OLIVEIRA ML, 2013).

Em outro estudo Malta DC, et al. (2017) descreveram a evolução anual da prevalência de excesso de peso e de obesidade na população adulta das capitais dos 26 estados brasileiros e no Distrito Federal entre 2006 e 2012. Vale ressaltar que a ocorrência do excesso de peso na população adulta das 27 cidades monitoradas pelo VIGITEL aumentou de 43,2\% (2006) para 51,0\% (2012), sendo de $1,37 \%$ o incremento anual médio calculado para o período. No entanto, a prevalência da obesidade aumentou de $11,6 \%$ para $17,4 \%$, com incremento anual de 0,89\%. Aumento estatisticamente significativo na prevalência do excesso de peso foi observado em todas as cidades, nos dois sexos, em todas as faixas etárias e em todos os níveis de escolaridade. Evolução semelhante foi observada para a obesidade.

Com relação ao gênero, $58 \%$ do sexo feminino e $42 \%$ do sexo masculino. Analisando esses mesmos indicadores por gênero, verifica-se que entre as mulheres a prevalência de sobrepeso e obesidade (I, II e III) foi de $27 \%$ e $13 \%$, respectivamente e, com relação aos homens, observa-se que a prevalência de sobrepeso e obesidade foi de $38 \%$ e 17 \% (GUERRA CG, 2016).

Para Cordeiro JYF e Freitas SRS (2016), a prevalência estimada do sobrepeso foi de $22,7 \%$ da população adulta, sendo maior nos indivíduos com mais idade, hipertensos e entre os homens com alto índice de gordura abdominal. 
Conforme Barroso TA, et al. (2017) a prevalência da obesidade nos estudos chama atenção, por se tratar de uma população relativamente jovem e que ainda é ativa, o que mostra o reflexo dos atuais hábitos alimentares no impacto a saúde. Portanto, evidenciou-se que $95 \%$ da amostra apresentaram obesidade abdominal, que é um importante fator de risco para DCV e outras comorbidades associadas.

\section{CONSIDERAÇÕES FINAIS}

O excesso de peso e a obesidade foram manifestados no grupo estudado, que teve maior acúmulo de gordura na região abdominal, associada a doenças como hipertensão arterial sistêmica e diabetes. Esses são fatores que preocupam, haja vista que estão associados ao risco do desenvolvimento de doenças cardiovasculares, aumento de complicações metabólicas e outros problemas de saúde. Diante desse contexto, evidenciou-se na pesquisa que há vários desafios na redução da taxa de crescimento da obesidade e do excesso de peso e as metas estabelecidas pelo Plano de Enfrentamento das Doenças Crônicas não Transmissíveis no Brasil e com isso, há necessidade de políticas efetivas por meio da prevenção, promoção da saúde pelos profissionais da saúde ao público mais vulnerável ao excesso de peso e a obesidade.

\section{REFERÊNCIAS}

1. ALMEIDA LM, et al. Estratégias e desafios da gestão da atenção primária à saúde no controle e prevenção da obesidade, Revista Eletrônica Gestão \& Saúde, Brasília, 2017; 8(1): 114-139.

2. BARROSO TA, et al. Associação Entre a Obesidade Central e a Incidência de Doenças e Fatores de Risco Cardiovascular. Int J Cardiovasc Sci. Rio de Janeiro, 2017; 30(5): 416-424.

3. BRASIL. Ministério da Saúde. Estratégias para o cuidado da pessoa com doença crônica: obesidade. Brasília- DF: Cadernos de Atenção Básica, n. 38. 2014.

4. BRASIL. Resolução CNS № 466, de 12 de dezembro de 2012, nos termos do Decreto de Delegação de Competência de 12 de novembro de 1991. CNS, 2012.

5. BRAVIN BM, et al. A influência do exercício físico na Obesidade infantil, Revista Ciência e Estudos Acadêmicos de Medicina, Mato Grosso, 2015; 1(4): 37-51.

6. CARVALHO CA, et al. Associação entre fatores de risco cardiovascular e indicadores antropométricos de obesidade em universitários de São Luís, Maranhão, Brasil. Ciência \& Saúde Coletiva, São Luís, 2015; 20(2): 479-490.

7. COSTA EC, et al. Evolução do excesso de peso e fatores associados em mulheres de 10 a 49 anos em Pernambuco, Nordeste, Brasil. Rev. Nutr, Campinas, 2014; 27(5): 513-524.

8. CORDEIRO JYF, FREITAS SRS. Prevalência e fatores associados ao excesso de peso em uma população urbana do interior do Amazonas, Brasil. Rev Bras Promoç Saúde, Fortaleza, 2016; 29(4): 533-543.

9. FREIRE RS, et al. Prática regular de atividade física: estudo de base populacional no norte de Minas Gerais, Brasil. Montes Claros, Rev Bras Med Esporte, Minas Gerais, 2014; 20(5): 345-349.

10. FLORÊNCIO RS, et al. Excesso ponderal em adultos jovens escolares: a vulnerabilidade da auto percepção corporal distorcida, Rer Bras Enferm, Fortaleza-CE, 2016; 2(69): 258-265.

11. GUERRA CG, et al. Prevalência de sobrepeso e obesidade em discentes de uma instituição de ensino superior da região macropolitana paulista. Saúde em Foco, São Paulo, n. 8, 2016.

12. LIMA NP, et al. Evolução do excesso de peso e obesidade até a idade adulta, Pelotas, Rio Grande do Sul, Brasil, 1982 2012. Cad. Saúde Pública, Rio de Janeiro, 2015; 31(9): 2017- 2015.

13. LOPES MS, et al. Limitantes da evolução ponderal de mulheres com excesso de peso após intervenção nutricional. $O$ Mundo da Saúde, São Paulo, 2016; 40(4): 433-443.

14. MALTA DC, et al. Prevalência e fatores associados com hipertensão arterial autorreferida em adultos brasileiros. Rev Saude Publica, São Paulo, 2017; 51(1): 1-11.

15. MAZUR, CE; NAVARRO, F. Insegurança alimentar e obesidade em adultos: Qual a relação? Saúde (Santa Maria), Santa Maria, 2015; 41(2): 35-44.

16. MELO TF, MELO TL. Estratégias de ensino utilizadas na educação física escolar para prevenção da obesidade em adolescentes escolares, Revista Ciências\&Ideias, Feira de Santana-BA, 2016; 7(3): 298-323. 
17. MIRANDA JMQ, et al. Prevalência de sobrepeso e obesidade infantil em instituições de ensino: públicas vs. Privadas, RevBrasMed Esporte, São Paulo, 2015; 21(2):104-107.

18. OLIVEIRA ML. Estimativa de custos da obesidade para o Sistema Único de Saúde do Brasil. Tese (Doutorado em Nutrição Humana) - Faculdade de Ciências da Saúde da Universidade de Brasília, Brasília, 2013; 109p.

19. SHOLER MC, et al. Exercícios físicos no combate ao sobrepeso e obesidade: Intensidade Versus Estresse Oxidativo, Rev. Ciência em Movimento, Rio Grande do Sul, 2016; 1(36): 71-85.

20. SHOMmER AV, et al. Excesso de Peso, Variáveis Antropométricas e Pressão Arterial em Escolares de 10 a 18 Anos, Porto Alegre (RS), Arq Bras Cardiol, Rio Grande do Sul, set. 2013.

21. TEIXEIRA RC, et al. Influências da mídia e das relações sociais na obesidade de escolares e a Educação Física como ferramenta de prevenção. Santa Cruz do Sul, Revista do Departamento de Educação Física e Saúde e do Mestrado em Promoção da Saúde da Universidade de Santa Cruz do Sul / Unisc, 2016; 17(2): 162-167. 